\title{
Citizen Relationship Management (CiRM): passado, presente e futuro de um conceito emergente
}

\author{
Daniel Carvalho ${ }^{1}$ \\ GISELA DEMO ${ }^{1}$ \\ JÚLIO MEDEIROS ${ }^{1}$ \\ FERNANDA SCUSSEL ${ }^{2}$ \\ ${ }^{1}$ UnIVERsidade de BrasílIa (UNB), BRAsílIa - DF, BRASIL \\ ${ }^{2}$ Universidade Federal de SANTA Catarina (UFSC), Florianópolis - SC, BRASIL
}

\section{Resumo}

Embora o Citizen Relationship Management (CiRM) tenha sido adotado mundialmente para aprimorar o relacionamento entre governos e cidadãos, pouco se sabe sobre o campo científico do CiRM. Este artigo relata uma revisão de literatura com o objetivo principal de analisar o campo científico do CiRM a fim de compreender sua estrutura intelectual e propor uma agenda de pesquisa. Analisamos 24 artigos publicados no período de 2005 a 2020, revelando que o CiRM é um conceito emergente na literatura científica, dependente do contexto e dividido em três categorias: o desenvolvimento de modelos integrativos; a relação entre governo e cidadãos; e a avaliação dos serviços públicos. A pesquisa sobre CiRM é predominantemente empírica, carecendo de maturidade conceitual que possibilite desenvolvimentos teóricos e empíricos contributivos. Com base nisso, propomos uma agenda de pesquisas abordando as principais lacunas: adoção, implementação e eficiência das estratégias de CiRM, além da integração dessas estratégias nos diferentes níveis de governo. Esta é a primeira revisão abrangente sobre CiRM, lançando luz sobre as bases do conhecimento de CiRM, compreendendo seu passado e o panorama atual. A partir disso, contribuímos com o desenvolvimento do corpo de conhecimento do CiRM, orientando os esforços de pesquisadores nas áreas de relacionamento entre governos e cidadãos; processos de adoção e implementação de CiRM; práticas de gestão pública; e eficiência na prestação de serviços públicos.

Palavras-chave: Gestão do Relacionamento com o Cidadão. Gestão do Serviço Público. Revisão de Literatura.

\section{Citizen Relationship Management (CiRM): the past, present, and future of an emerging concept}

\begin{abstract}
Although Citizen Relationship Management (CiRM) has been adopted worldwide to enhance the relationship between governments and citizens, little is known about the scientific field of CiRM. This article uses a literature review to analyze the scientific field of CiRM to understand its intellectual structure and set a research agenda. We analyzed 24 articles published from 2005 to 2020 , revealing that $\mathrm{CiRM}$ is an emergent concept in scientific literature, context-dependent, and divided into three categories: the development of integrative models; the relationship between government and citizens; and the evaluation of public services. Research on CiRM is predominantly empirical, lacking the conceptual maturity that enables contributive theoretical and empirical developments. We propose a research agenda addressing the main gaps: adoption, implementation, and efficiency of CiRM strategies, as well as the integration of such strategies in different government levels. This is the first comprehensive review about CiRM, shedding light on the knowledge of CiRM, understanding its past, and the current panorama. We contribute to the development of CiRM knowledge, guiding the researchers' efforts in the fields of relationship between governments and citizens; CiRM adoption and implementation processes; public managerial practice; and efficiency in public service provision.
\end{abstract}

Keywords: Citizen Relationship Management. Public Sector Management. Literature Review.

\section{Citizen Relationship Management (CiRM): pasado, presente y futuro de un concepto emergente}

\section{Resumen}

Aunque el Citizen Relationship Management (CiRM) ha sido adoptado en todo el mundo para mejorar la relación entre gobiernos y ciudadanos, se sabe poco sobre el campo científico del CiRM. Este artículo reporta una revisión de la literatura con el objetivo principal de analizar el campo científico del CiRM para comprender su estructura intelectual y establecer una agenda de investigación. Analizamos 24 artículos, publicados entre 2005 y 2020, que revelan que el CiRM es un concepto emergente en la literatura científica, dependiente del contexto y dividido en tres categorías: el desarrollo de modelos integradores; la relación entre gobierno y ciudadanos; y la evaluación de los servicios públicos. La investigación sobre CiRM es predominantemente empírica y carece de una madurez conceptual que permita desarrollos teóricos y empíricos contribuyentes. Sobre esa base, proponemos una agenda de investigación que aborde las principales brechas: adopción, implementación y eficiencia de las estrategias del CiRM, además de la integración de dichas estrategias en los diferentes niveles de gobierno. Esta es la primera revisión integral sobre CiRM que arroja luz sobre sus bases de conocimiento y entiende su pasado y el panorama actual. A partir de esto, contribuimos con el desarrollo del cuerpo de conocimiento del CiRM, orientando los esfuerzos de los investigadores en los campos de relación entre gobiernos y ciudadanos; procesos de adopción e implementación de CiRM; prácticas de gestión pública; y eficiencia en la prestación de servicios públicos.

Palabras clave: Gestión de relaciones ciudadanas. Gestión de servicios públicos. Revisión de literatura. 


\section{INTRODUÇÃO}

O surgimento da Nova Gestão Pública, que mudou a dinâmica da prestação de serviços públicos, com base nas limitações do Modelo Burocrático e com sucesso implantado na Grã-Bretanha, Estados Unidos, Austrália e Nova Zelândia (Abrucio, 2007), novas rotas para o desenvolvimento de uma gestão pública estratégica foram criadas. Naquela época, duas grandes preocupações nortearam as transformações no setor público, levando os governos a redefinirem suas atribuições em todo o mundo: a crise do papel do Estado na década de 1980 e a globalização da economia (Bresser-Pereira, 1996; Lara \& Gosling, 2016a).

Desde então, o mundo passou por diversas reformas administrativas que deram origem a novos discursos e práticas inspiradas no setor privado, que se tornou referência para as instituições públicas em diferentes níveis de governo. Nesse sentido, a Administração Pública passou a implementar mecanismos de produtividade, ações orientadas para o serviço, descentralização administrativa, eficiência na prestação de serviços, melhoria dos serviços, mercadologia e accountability (Secchi, 2009). Tratam-se de esforços para simplificar o acesso à informação e aos serviços públicos oferecidos aos cidadãos, empresas e servidores públicos, melhorando a qualidade do serviço e gerando redução de custos (Lara \& Gosling, 2016a; Ponte, 2015).

Esse cenário passou a ser investigado cientificamente, engendrando o conceito de Citizen Relationship Management (CiRM), ou Gestão do Relacionamento com o Cidadão, uma filosofia gerencial que representa o esforço de todos os níveis de governo em atender com rapidez e precisão às necessidades, solicitações e indagações dos cidadãos, promovendo mudanças nas políticas, práticas e procedimentos do setor público (Shaikh \& Khan, 2014).

As raízes do CiRM é Customer Relationship Management (CRM), ou Gestão do Relacionamento com o Consumidor, uma filosofia de negócios que surgiu na década de 1990 para estabelecer, manter e melhorar os relacionamentos entre clientes e organizações, integrando tecnologia, pessoas e processos (Payne, 2012; Payne \& Frow, 2006). Apesar das semelhanças entre os modelos privado e público, o CRM aposta na promoção de benefícios relacionais para empresas e clientes, enquanto o CiRM privilegia a prestação de serviços que são direitos dos cidadãos, contribuindo para o desenvolvimento da cidadania e integração da sociedade (Demo \& Pessôa, 2015; Fleury, 2005; Peci, Pieranti \& Rodrigues, 2008). Os cidadãos são obrigados, em certa medida, a usar serviços que são monopolizados pelos governos, enquanto no setor privado o objetivo é maximizar os lucros com base na atração e fidelidade do cliente (Keramati, Saremi \& Mofrad, 2011). Isso significa que as estratégias do CiRM devem abordar a complexidade da arena governamental, os interesses políticos, os diferentes momentos das práticas públicas e as demandas crescentes da sociedade (Tavana, Zandi \& Katehakis, 2013). Portanto, embora o CiRM seja derivado do CRM, ele possui particularidades próprias, exigindo a devida análise de seu campo científico.

O objetivo do CiRM é ajudar os gestores públicos a enfrentar os desafios relativos à prestação de serviços, questões de prestação de contas e interação com os cidadãos, empresas privadas e outros atores sociais que intermediam a relação entre governo e sociedade (Larsen \& Milakovich, 2005). A ideia de melhorar a Administração Pública configurou uma agenda de pesquisas em todo o mundo, abrangendo estudos sobre ouvidoria governamental (Lara \& Gosling, 2016a); satisfação dos cidadãos (Ghodousi, Alesheikh \& Saeidian, 2016); percepção sobre o relacionamento com o cidadão (Demo \& Pessôa, 2015); adoção e implantação do CiRM (Eslami, 2012; Duque, Varajão, Vitor \& Dominguez, 2013; Keramati et al., 2011); e modelos de CiRM (Al-Khouri, 2012; Lara \& Gosling, 2016b; Pollard, Young \& Gregg, 2006).

Em suma, o CiRM tem se tornado relevante entre as organizações públicas como alternativa para enfrentar desafios em termos de representatividade, legitimação do Estado e oferta de serviços públicos nas sociedades de consumo. No entanto, os avanços neste campo científico mostram dificuldades na adoção e implementação do CiRM (Andrade, 2013; Ponte, 2015), sem um claro entendimento de sua conceituação, funcionamento e consequências, o que pode ser justificado pela inovação que o CiRM significa em termos de práticas de gestão pública (Demo \& Pessôa, 2015). Algumas questões surgem desta discussão: como a literatura sobre CiRM se desenvolveu ao longo do tempo? Como seu corpo de conhecimento está organizado atualmente? Quais são os caminhos para o futuro da pesquisa CiRM?

Não foram identificados trabalhos dedicados à compreensão do CiRM como um campo científico, o que acreditamos ser um passo importante para o desenvolvimento teórico e empírico desse conceito emergente. Assim, este artigo relata uma revisão de literatura com o objetivo principal de analisar o campo científico do Citizen Relationship Management (CiRM) a fim de compreender sua estrutura intelectual e definir uma agenda de pesquisa. Segundo Tranfield, Denyer e Smart (2003), as revisões de literatura são um importante tipo de estudo para o desenvolvimento de tópicos sob a égide da pesquisa em 
gestão, uma vez que englobam diferentes abordagens em uma síntese, proporcionando um entendimento mais amplo de um tópico específico a partir da análise de estudos primários. Além disso, a análise da estrutura intelectual de um determinado campo fornece informações sobre os artigos, periódicos, autores e temas emergentes mais influentes, permitindo traçar futuras direções de investigação (Zupic \& Carter, 2015). Por fim, as revisões de literatura contribuem para o estudo de campos emergentes (Templier \& Paré, 2015; Zupic \& Carter, 2015), que é o caso do CiRM.

Até onde se sabe, esta é a primeira revisão abrangente sobre CiRM, um conteúdo de grande interesse social que vem crescendo tanto na prática de gestão pública quanto no desenvolvimento acadêmico. Diante disso, lançamos luz sobre as bases do conhecimento do CiRM, entendendo seu passado e como chegamos ao panorama atual: um conceito emergente que vem sendo subteorizado, considerando a relevância do tema para a sociedade. A maior parte da pesquisa sobre CiRM é empírica, carecendo da maturidade conceitual que irá promover desenvolvimentos empíricos. A compreensão das lacunas desse campo científico nos conduziu à proposição de uma agenda de pesquisas para investigações futuras, contribuindo para a construção teórica e empírica desse conceito emergente. Além disso, os resultados apresentados neste trabalho funcionam como um diagnóstico para que os atores governamentais responsáveis pela prática gerencial explorem as possibilidades de relacionamento com os cidadãos, valorizando suas ações e promovendo uma sociedade melhor.

Após esta introdução, apresentamos uma síntese do referencial teórico do qual emerge o conceito de CiRM, seguida da descrição dos procedimentos metodológicos e dos achados. A próxima seção discute os resultados e propõe uma agenda de pesquisa. Conclusões e referências encerram o artigo.

\section{REFERENCIAL TEÓRICO}

A relação entre cidadãos e governos tornou-se uma mudança estratégica na Administração Pública moderna, contexto em que a interação entre esses atores sociais surge como o vetor para a melhor prestação do serviço público e para o alcance da eficiência nas instituições públicas (Ponte, 2015; Secchi , 2009). Esta mudança se assenta em uma relação mais dinâmica em que os cidadãos prestam mais informações à Administração Pública através de diferentes canais - plataformas online, call-centers e agências físicas, seguida da utilização dessas informações para desenvolver um melhor serviço público (Demo \& Pessôa , 2015; Lam, 2005; Larsen \& Milakovich, 2005; Lytras, 2006; Schellong, 2008). Nesse sentido, o Citizen Relationship Management (CiRM) surge como um conjunto de práticas gerenciais voltadas ao desenvolvimento do relacionamento entre os cidadãos e os governos, gerando benefícios relacionais para ambos os atores.

A adoção de estratégias do setor privado para melhorar a prestação de serviços públicos tem norteado a adaptação de estratégias de CRM ao setor público, nas quais o CiRM surge com o objetivo de estabelecer e desenvolver uma relação entre as organizações da gestão pública e os usuários dos serviços públicos (Demo \& Pessôa, 2015; Kannabiran, Xavier \& Anantharaaj, 2004; Ponte, 2015). Nesta perspectiva, o CiRM ajuda a melhorar o atendimento, a qualidade na entrega, a eficiência e a atender às reais necessidades do cidadão. Além disso, contribui para a promoção da cidadania e para a melhoria da imagem/ reputação dos serviços públicos em geral (Demo \& Pessôa, 2015; Schellong, 2008).

No âmbito público, o CiRM surge como uma solução para gerenciar as complexas interações entre cidadãos e governos, permitindo a integração de serviços, pessoas, práticas, processos e tecnologias, mantendo o propósito de desenvolvimento social (Kavanagh, 2007). O cidadão agora pode interagir com os órgãos públicos por meio de diversos canais como sites, balcão de atendimento, correio, call center ou atendimento presencial, onde cada interação resultará em uma experiência de atendimento diferente. Nesse sentido, o CiRM contribui para mudar a abordagem de auto-referência dos governos para uma estratégia centrada no cidadão (Al-Khouri, 2012; Fil'a, Schwarczová \& Mura, 2015; La Falce, De Muylder, Pressot \& Toivanen, 2015; Zamanian, Khaji e Emamian, 2011). Na medida em que os autores convergem, o Estado busca uma visão holística dos ciclos de formulação de políticas públicas, a fim de obter legitimidade de lideranças, resultados de eficácia ou gerar consenso em termos de visão de governo.

A mudança da abordagem governamental, em que o cidadão é considerado cliente dos serviços públicos, tem sido apontada como um divisor de águas no processo de implementação do CiRM na Administração Pública, embora os cidadãos tenham uma relação multifacetada com o governo que vai além a ideia de ser um consumidor regular do serviço (Demo \& Pessôa, 2015; Kavanagh, 2007). Esta nova percepção junto aos cidadãos incentivou os entes públicos a melhor atender às demandas 
dos serviços e a implementar modelos de CiRM para reduzir custos operacionais, gerir reclamações e reduzir o tempo de resposta. Por outro lado, os cidadãos são mais pró-ativos, exigindo qualidade e rapidez na prestação do serviço público (Demo \& Pessôa, 2015). Portanto, a Administração Pública deve preocupar-se com a agilidade, regularidade, consistência e disponibilidade dos serviços públicos em todos os canais de atendimento ao cidadão (Fil'a et al., 2015).

Nesse contexto, o uso da tecnologia da informação tem promovido a ampliação da interação entre governos e seus cidadãos, incentivado a transparência e orientado gestores públicos com informações sobre a percepção dos cidadãos quanto ao seu relacionamento com os órgãos de serviços públicos (Demo \& Pessôa, 2015; Lara \& Gosling, 2016b, Zamanian et al., 2011). Nesse cenário, o governo eletrônico (e-government) surge como uma importante ferramenta para a interação entre órgãos públicos e cidadãos (Larsen \& Milakovich, 2005), utilizando a internet como elemento fundamental na construção da governança (Lytras, 2006). Descobertas recentes mostram que o governo eletrônico é o principal parceiro da governança e da transparência, pois melhora a eficiência administrativa, promove o comportamento ético e aumenta a confiança da sociedade e a convicção no governo (Twizeyimana \& Andersson, 2019).

De acordo com Kavanagh (2007) e Ponte (2015), uma estratégia de CiRM de sucesso só é alcançada por funcionários públicos comprometidos em prestar um bom serviço e por meio de processos que gerem valor público para os cidadãos. Ponte (2015) explica que a Administração Pública deve alinhar as estratégias às necessidades dos cidadãos, integrando ferramentas tecnológicas com desenho organizacional e servidores públicos. No entanto, a implementação parece ser o ponto crítico para o CiRM, uma vez que os governos não são capazes de dar respostas rápidas às demandas e necessidades dos serviços públicos (Andrade, 2013; Ponte, 2015). Conforme proposto em Demo e Pessôa (2015), a adoção e implementação do CiRM está ligada a uma abordagem centrada no cidadão, tal como o CRM coloca o cliente no centro das estratégias organizacionais.

Com base nesse referencial teórico, entendemos que CiRM é um corpo de conhecimento em construção. A literatura do CiRM tem muito a aprender com as práticas do setor privado e seus casos de sucesso, no entanto, é fundamental abraçar as complexidades da arena pública a fim de orientar futuros desenvolvimentos, academicamente e na prática gerencial.

\section{PROCEDIMENTOS METODOLÓGICOS}

Uma revisão de literatura foi conduzida seguindo as orientações de Templier e Paré (2015) e seus procedimentos para a realização de revisões de literatura, divididos em seis atividades: (i) formulação do problema, (ii) pesquisa bibliográfica, (iii) triagem para inclusão, (iv) ) avaliação da qualidade, (v) extração de dados e (vi) análise e síntese de dados.

Na primeira etapa, formulação do problema, Templier e Paré (2015) afirmam que os autores devem justificar a necessidade de uma revisão da literatura, abordar a questão de pesquisa e o objetivo do estudo e informar como o estudo foi desenhado. Acreditamos que a introdução deste artigo atende aos requisitos relativos à questão de pesquisa e à formulação do objetivo. No que se refere ao desenho desta revisão de literatura, optou-se por combinar dois métodos de análise de dados secundários, recorrendo a uma análise de revisão sistemática qualitativa (Tranfield et al., 2003) e uma análise bibliométrica (Zupic \& Carter, 2015).

De acordo com Tranfield et al. (2003), as revisões sistemáticas auxiliam os pesquisadores a organizar o conhecimento sobre um determinado tema e a tirar conclusões sobre o fenômeno. No entanto, explicam que a natureza qualitativa das revisões sistemáticas em ciências da administração está associada a um nível mais alto de subjetividade. Para evitar esse viés, combinamos a revisão sistemática com a abordagem quantitativa da análise bibliométrica e seu potencial para elevar a transparência e reprodutibilidade do processo de revisão (Zupic \& Carter, 2015). Como afirmam os autores, a análise bibliométrica fornece resultados baseados em dados bibliográficos de outros estudos primários, permitindo conclusões sobre a estrutura de um campo, suas redes sociais e percepções sobre temas de maior interesse.

A segunda etapa proposta por Templier e Paré (2015) é a busca na literatura. Isso significa que, antes de selecionar os estudos primários a serem avaliados na revisão da literatura, os autores devem explorar a literatura da área em investigação, o que foi feito na introdução deste artigo. Essa ação é seguida da seleção de uma população de estudos e da descrição da estratégia de busca, procedimentos e fontes de dados. 
A coleta de dados foi realizada em julho de 2020 nas bases de dados científicas Web of Science e Scopus, as duas fontes mais comuns de dados bibliográficos (Chadegani et al., 2013; Zupic \& Carter, 2015). Para a seleção dos artigos, foram utilizadas as palavras-chave "CzRM", "CiRM" e "Citizen Relationship Management", com o operador booleano "OR". Os pesquisadores optaram por não limitar o período de análise, visto que o tema da pesquisa é novo. Os filtros de busca utilizados foram "artigos" como tipo de publicação; e Gestão, Negócios e Administração Pública para a área de conhecimento.

Na etapa de triagem para inclusão, identificamos 12 artigos da Web of Science e 20 artigos da Scopus. Analisamos o título e o resumo dos estudos primários, além da exclusão de duplicados, chegando a um corpus de 24 artigos. Decidimos manter todos os estudos devido ao fato de o CiRM ser um conceito emergente. Depois, conforme proposto por Templier e Paré (2015), a qualidade dos artigos foi avaliada. Nesse sentido, nos dedicamos à leitura do artigo na íntegra, sendo que todos os 24 artigos foram mantidos, gerando a amostra final deste estudo.

Na fase de extração dos dados, acessamos ano de publicação, autoria, periódico de publicação e país de publicação, recorrendo a um software bibliométrico para gerar a análise, que inclui uma análise de acoplamento bibliográfico, conforme indicado por Zupic e Carter (2015). O VOSviewer 1.6.11 foi utilizado devido à sua capacidade em proporcionar relações cooperativas dentro de um campo científico, possibilitando a elaboração do acoplamento bibliográfico (Van Eck \& Waltman, 2017). Também analisamos o número de citações dos artigos, usando dados do Google Scholar em julho de 2020.

A etapa final é a análise e síntese dos dados, momento em que Templier e Paré (2015) sugerem aos autores que organizem, comparem e interpretem os dados de forma a criar a contribuição da literatura para o corpo de conhecimento sob investigação.

\section{RESULTADOS}

Os primeiros resultados referem-se à apresentação dos 24 artigos que compuseram nossa amostra, resumidos no Quadro 1.

\section{Quadro 1 \\ Artigos sobre CiRM}

\begin{tabular}{|c|c|}
\hline Artigo & Referência \\
\hline Citizen Relationship Management and E-government & Larsen e Milakovich (2005) \\
\hline Integration challenges towards increasing e-government maturity & $\operatorname{Lam}(2005)$ \\
\hline $\begin{array}{l}\text { The semantic electronic government: Knowledge management for citizen } \\
\text { relationship and new assessment scenarios }\end{array}$ & Lytras (2006) \\
\hline $\begin{array}{l}\text { Reengineering municipality citizen electronic complaint system through citizen } \\
\text { relationship management }\end{array}$ & Chu et al. (2008) \\
\hline $\begin{array}{l}\text { Creating public value in e-government: A public-private-citizen collaboration } \\
\text { framework in Web } 2.0\end{array}$ & Hui e Hayllar (2010) \\
\hline $\begin{array}{l}\text { The value chain of citizen relationship management (CzRM): A framework for } \\
\text { improvement }\end{array}$ & Zamanian et al. (2011) \\
\hline $\begin{array}{l}\text { Citizen relationship management critical success factors: An empirical study of } \\
\text { municipality of Tehran }\end{array}$ & Keramati et al. (2011) \\
\hline A comprehensive conceptual framework for the E-government realization & $\begin{array}{l}\text { Dehkordi, Sarlak, } \\
\text { Pourezzat e Ghorbani (2012) }\end{array}$ \\
\hline $\begin{array}{l}\text { A study on the customer relationship management model adaptability with the } \\
\text { municipality services and duties environment }\end{array}$ & Eslami (2012) \\
\hline $\begin{array}{l}\text { A hybrid fuzzy group ANP-TOPSIS framework for assessment of e-government } \\
\text { readiness from a CiRM perspective }\end{array}$ & Tavana et al. (2013) \\
\hline Implementation of CRM systems in Portuguese Municipalities & Duque et al. (2013) \\
\hline $\begin{array}{l}\text { FTiS: A new model for effective urban management: A case study of urban } \\
\text { systems in Iran }\end{array}$ & $\begin{array}{l}\text { Akhondzadeh-Noughabi, } \\
\text { Alizadeh, Ahmadvand e } \\
\text { Minaei-Bidgoli (2013) }\end{array}$ \\
\hline
\end{tabular}




\begin{tabular}{|c|c|}
\hline Artigo & Referência \\
\hline $\begin{array}{l}\text { CRM in the public management: Development and validation of a Citizen } \\
\text { Relationship Scale (CiRS) }\end{array}$ & Demo e Pessôa (2015) \\
\hline $\begin{array}{l}\text { Citizen satisfaction survey as a tool of citizen relationship management of local } \\
\text { government in Slovakia }\end{array}$ & Fil'a et al. (2015) \\
\hline $\begin{array}{l}\text { Concerns Management, E-Government and E-Participation: Experiences and } \\
\text { Findings from Germany }\end{array}$ & $\begin{array}{l}\text { Vaerst, Steffens e } \\
\text { Lokaiczyk (2015) }\end{array}$ \\
\hline $\begin{array}{l}\text { Analyzing public participant data to evaluate citizen satisfaction and to prioritize } \\
\text { their needs via K-means, FCM and ICA }\end{array}$ & Ghodousi et al. (2016) \\
\hline Factor analysis that make up the ombudsman on relations with the citizen & Lara e Gosling (2016a) \\
\hline $\begin{array}{l}\text { Systems Administration in Ontology-Based Applications: The Case of Citizen } \\
\text { Relationship Management }\end{array}$ & Alexandrova et al. (2016) \\
\hline $\begin{array}{l}\text { Citizen Relationship Management System Users' Contact Channel Choices: } \\
\text { Digital Approach or Call Approach? }\end{array}$ & Wu (2017) \\
\hline $\begin{array}{l}\text { Evaluating Citizen Satisfaction and Prioritizing their Needs Based on Citizens } \\
\text { Complaint Data }\end{array}$ & $\begin{array}{c}\text { Ghodousi, Alesheikh, } \\
\text { Saeidian, Pradhan } \\
\text { e Lee (2019) }\end{array}$ \\
\hline $\begin{array}{l}\text { Citizen Feedback and Executives Improving Government Performance Through } \\
\text { Citizen Relationship Management }\end{array}$ & Havaeji e Albadvi (2019) \\
\hline $\begin{array}{l}\text { Gamification Strengthening the Relationship Between the Government and the } \\
\text { Citizens }\end{array}$ & Wang e riyanto (2019) \\
\hline $\begin{array}{l}\text { Citizen Relationship Management CNRM to Build the Awareness of Anticorruption } \\
\text { Collaborative Governance Perspective }\end{array}$ & $\begin{array}{l}\text { Sukarno, Azizah, } \\
\text { Nawangsari, Izaak } \\
\text { e Farida (2020) }\end{array}$ \\
\hline $\begin{array}{l}\text { Does Citizens } 311 \text { System Use Improve Satisfaction with Public Service } \\
\text { Encounterslessons for Citizen Relationship Management }\end{array}$ & Wu (2020) \\
\hline
\end{tabular}

Fonte: Elaborado pelos autores.

A primeira análise dos resultados indica que os artigos Citizen Relationship Management and E-government (Larsen \& Milakovich, 2005) e Integration challenges towards increasing e-government maturity (LAM, 2005) são os artigos seminais sobre CiRM, uma vez que são os artigos mais antigos nesse tema, considerando os parâmetros da busca realizada.

Larsen e Milakovich (2005) discutem a introdução do CiRM na esfera pública como estratégia para responder às demandas da população em relação à maior qualidade dos serviços. Os autores discutem a adaptação do CRM ao CiRM, abordando particularmente a transformação dos cidadãos em consumidores e seus efeitos na governança democrática e na administração pública. Eles afirmam que essa mudança de cidadãos para consumidores empoderou a sociedade e representa uma mudança na relação entre governos e cidadãos, que deve ser administrada de maneira adequada. Nesse contexto, o CiRM surge como uma estratégia viável para aprimorar esse relacionamento e ajudar os gestores públicos a enfrentar os desafios desse novo e complexo contexto.

Lam (2005) apresenta o CiRM como uma perspectiva importante para enfrentar os desafios do governo na sociedade contemporânea, esclarecendo o papel do governo eletrônico na promoção do relacionamento entre governos e cidadãos. $\mathrm{O}$ autor propôs um modelo de governo eletrônico composto por quatro estágios progressivos: (i) governo eletrônico informativo, (ii) governo eletrônico transacional, (iii) governo eletrônico integrado de processos e (iv) governo eletrônico integrado de serviços. $\mathrm{O}$ artigo diferencia o governo eletrônico do CiRM, mostrando que o governo eletrônico é uma estratégia de CiRM que proporcionará melhores resultados quando integrada a outras práticas de CiRM, como processos colaborativos e integração de tecnologia.

A análise desses dois artigos seminais justifica uma das observações que fizemos durante a leitura completa dos 24 artigos: há uma confusão conceitual entre CiRM e governo eletrônico. CiRM é um conceito mais amplo, uma filosofia empresarial holística trazida ao setor público para melhorar a gestão da relação entre governos e cidadãos, enquanto o governo eletrônico é o uso em aplicativos da internet para fornecer acesso e entrega de informações governamentais e serviços públicos (Larsen \& Milakovich, 2005). Assim, o governo eletrônico pode ser considerado uma estratégia do CiRM. 
Considerando 2005 como o início do campo científico do CiRM, o passo seguinte foi avaliar o desenvolvimento do campo em termos de produção científica, conforme ilustra a Figura 1.

Figura 1

Número de artigos publicados sobre CiRM

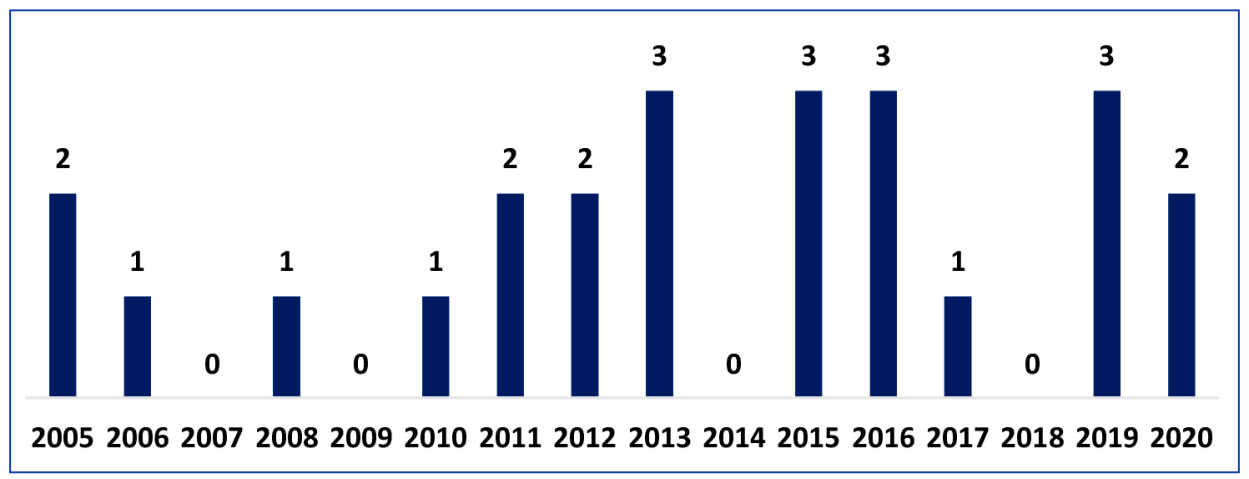

Fonte: Elaborada pelos autores.

De 2005 a 2020, 24 artigos foram publicados em um padrão relativamente estável. Os anos de 2013, 2015, 2016 e 2019 foram os mais produtivos, com três artigos por ano. A partir disso, entendemos o Citizen Relationship Management - CiRM como um tema em desenvolvimento, já que a primeira publicação identificada foi em 2005.

Dos 24 artigos, nove eram artigos conceituais e 15 eram pesquisas empíricas. A maioria dos trabalhos conceituais foram dedicados ao desenvolvimento de quadros conceituais para entender o papel da gestão do conhecimento no contexto do CiRM (Lytras, 2006), para criar um modelo de adaptabilidade dos serviços públicos (Eslami, 2012) e para abordar modelos de governo eletrônico (Dehkordi et al., 2012; Hui \& Hayllar, 2010). A abordagem teórica também é utilizada para explorar a administração de sistemas (Alexandrova et al., 2016), para investigar o papel do CiRM nas estratégias anticorrupção (Sukarno et al., 2020) e para analisar a cadeia de valor que compõe o CiRM (Zamanian et al. , 2011). A partir disso, identificamos um grande esforço na construção de um framework para o CiRM, porém, pouca atenção é direcionada às conceituações do construto ou à investigação de seus antecedentes e consequentes. Esta poderia ser uma teorização importante para validar os modelos de CiRM em pesquisas empíricas.

A primeira pesquisa empírica identificada foi o trabalho de Chu, Yeh e Chuang (2008), um estudo de caso em Taiwan. Também identificamos estudos de caso na Alemanha (Vaerst et al., 2015), Irã (Akhondzadeh-Noughabi et al., 2013), Portugal (Duque et al., 2013) e Teerã (Keramati et al., 2011). A literatura abrange a análise de cluster para compreender a satisfação do cidadão (Ghodousi et al., 2016) e as queixas dos cidadãos (Ghodousi et al., 2019). Também observamos pesquisas explorando o feedback dos cidadãos sobre o desempenho do governo (Havaeji \& Albadvi, 2019), o papel da ouvidoria (Lara \& Gosling, 2016a), a preferência dos cidadãos na abordagem digital ou por telefone (Wu, 2017) e a satisfação dos cidadãos (Fil'a et al., 2015; Wu, 2020). Destacamos o artigo de Demo e Pêssoa (2015) e a proposição da primeira escala científica validada para mensurar a percepção de relacionamento do cidadão, abordando os principais aspectos da relação entre governo e cidadãos.

Esses achados mostram que o conhecimento acadêmico sobre o CiRM é construído a partir da necessidade de cada contexto de investigação, uma vez que não há uma conexão clara entre o tema dos artigos: eles parecem servir à prática gerencial dos contextos investigados. Percebemos que a literatura ainda é exploratória. Compreendemos que o CiRM ainda é um conceito em construção, e a falta de conceituações e instrumentos de mensuração são o próximo passo para o desenvolvimento teórico e empírico desse conceito emergente.

A seguir, identificamos os periódicos que publicaram artigos sobre CiRM no período analisado. Essas ações mostraram 21 periódicos publicando sobre CiRM. Considerando que temos um total de 24 artigos espalhados em 21 periódicos, reforçamos a ideia de que CiRM é um construto em desenvolvimento. Entendemos que um novo tema desperta o interesse de muitos pesquisadores e periódicos científicos, justificando a falta de concentração das publicações em um determinado periódico. As revistas Australian Journal of Public Administration, Cities e Eletronic Government publicaram 2 artigos cada, e os outros 18 periódicos publicaram um artigo cada. 
Posteriormente, identificamos os 5 periódicos mais citados, usando dados do Google Scholar em julho de 2020. Segundo Zupic e Carter (2015), o número de citações é uma métrica de influência de um periódico ou artigo, indicando que o conteúdo dos periódicos/artigos mais citados são importantes para o desenvolvimento do conhecimento em uma determinada área. Assim, o periódico mais influente na literatura de CiRM é o Australian Journal of Public Administration (80 citações), seguido por Eletronic Government (45 citações), Information and Management (37 citações), Cities (14 citações) e o Journal of E-Government ( 10 citações).

A seguir, analisamos os artigos por país de publicação, a fim de revelar se houve concentração no desenvolvimento do conhecimento. O Irã foi o país com o maior número de artigos publicados sobre CiRM (7 artigos), seguido por Taiwan (3 artigos) e Brasil, China e Indonésia com 2 artigos cada. Os demais países identificados publicaram apenas um artigo no período analisado. Nenhuma rede de pesquisa foi identificada. O número de países que publicam sobre CiRM pode ser um indicador do interesse dos governos em adotar e implementar estratégias de CiRM para enfrentar os desafios de produzir um serviço público eficiente. Este interesse global, apesar da falta de uma rede estruturada de pesquisa, lança luz sobre o fato de que o CiRM é um construto dependente do contexto. Ou seja, depende do contexto de análise, das particularidades de cada país, estado, região ou cidade para ter sucesso. O cenário político, as condições sociais e econômicas, a cultura e a dinâmica entre governo, cidadãos e atores sociais devem ser acessados na formulação de uma estratégia de CiRM.

Em seguida, foram analisados os artigos mais influentes, utilizando o critério de número de citações (Zupic \& Carter, 2015). Acessamos o número de citações no Google Scholar em julho de 2020.

$\mathrm{O}$ artigo mais citado na literatura de CiRM desde 2005 foi Creating public value in e-government: A public-private-citizen collaboration framework in Web 2.0, escrito por Hui e Hayllar (2010), com 152 citações. Os autores enfocam os desafios enfrentados pelos gestores públicos na tentativa de inovar na prestação de serviços públicos, contexto em que CiRM é visto como uma ferramenta que possibilita interações colaborativas entre governo, cidadãos e partes interessadas privadas. A implementação do CiRM está ligada ao uso crescente da Web 2.0 na criação de conteúdo para o público e design de serviços, na tentativa de acessar informações do cidadão e aumentar o valor da entrega de serviços públicos.

O segundo lugar no ranking é compartilhados pelos artigos Citizen Relationship Management and E-government (Larsen \& Milakovich, 2005) e A hybrid fuzzy group ANP-TOPSIS framework for assessment of e-government readiness from a CiRM perspective (Tavana et al., 2013), com 52 citações cada.

O artigo de Larsen e Milakovich (2005) é o artigo seminal na literatura CiRM e apresenta aos estudos de gestão pública uma alternativa trazida pelo contexto das empresas privadas na tentativa de ajudar os gestores públicos a lidar com os problemas da prestação de serviços públicos e as demandas da sociedade. O CiRM surge como uma alternativa de desafio-solução no cenário do setor público contemporâneo, sendo uma ferramenta para aprimorar a comunicação, promover melhores serviços e reduzir custos públicos. Por sua vez, a publicação de Tavana et al. (2013) propõe um modelo de avaliação de prontidão governamental, que significa a capacidade dos governos em acessar as informações dos cidadãos e propor soluções aos problemas públicos de forma ágil, utilizando a internet como principal ferramenta.

Na sequência, Lytrs (2006) escreveu The semantic electronic government: Knowledge management for citizen relationship and new assessment scenarios, no qual o autor conceitua o CiRM como uma tarefa intensiva em conhecimento, baseada em infraestruturas de conhecimento, fluxos de conhecimento e transformações dinâmicas ( 35 citações). 0 objetivo principal do artigo é lançar luz sobre a gestão do conhecimento convergente na adoção do CiRM. O autor afirma que a complexidade em torno da diversidade de estruturas, regulamentos, procedimentos e a natureza da oferta pública afetam a dinâmica da gestão do conhecimento, uma integração fundamental para o sucesso da implementação do CiRM.

O quarto artigo mais citado foi Reengineering municipality citizen electronic complaint system through citizen relationship management (Chu, Yeh \& Chuang, 2008), com 24 citações. Este trabalho enfatiza o papel do governo eletrônico e sua função principal de possibilitar a interação entre o governo e os cidadãos. Segundo os autores, o governo eletrônico é o principal insumo da estratégia do CiRM, pois permite a comunicação entre os atores e, a partir disso, melhor entendimento das demandas dos cidadãos, melhoria da qualidade dos serviços e participação pública.

Por fim, em CRM in the public management: Development and validation of a Citizen Relationship Scale (CiRS), Demo e Pessôa (2015) estabeleceram um marco na literatura CiRM ao desenvolver e validar uma escala científica para mensurar a percepção dos cidadãos em relação às iniciativas de CiRM na gestão pública (19 citações). A escala pode ser utilizada para validações em 
diferentes contextos, respeitando a complexidade de cada cenário, revelando os aspectos mais relevantes na relação entre governos e cidadãos. Nesse sentido, os resultados de estudos que utilizarem a escala podem ser utilizados como ferramenta de diagnóstico por gestores públicos para promover uma gestão eficaz do relacionamento com os cidadãos.

Ao analisar os cinco artigos mais citados, há uma convergência entre eles quanto aos desafios de replicar as estratégias do setor privado na complexidade dos governos, contexto formado por diferentes estruturas, instituições, interesses e questões políticas, além das necessidades dos cidadãos e da sociedade como um todo. Prevemos, a partir dos estudos, que os gestores públicos estão apresentando uma postura diferenciada em suas ações como servidores públicos, atuando de forma inovadora com o objetivo de prestar um serviço de melhor qualidade em um prazo razoável. A comunicação, a interação e a prontidão dos governos são conteúdos que permeiam os artigos mais citados, revelando que este não é apenas um assunto de interesse público, mas um referencial que necessita de maiores desenvolvimentos na pesquisa acadêmica.

A última etapa foi explorar as principais discussões na literatura sobre CiRM. Criamos um acoplamento bibliográfico a fim de abordar os principais subcampos de pesquisa em CiRM. Segundo Zupic e Carter (2015), o acoplamento bibliográfico é uma representação visual da conexão entre referências, utilizada como medida de semelhança, criando clusters que reúnem interesses similares. Devido ao baixo número de artigos publicados no período analisado (24 artigos), o acoplamento bibliográfico considerou o compartilhamento de pelo menos um artigo. Identificamos três clusters, como ilustra a Figura 2.

Figura 2

Acoplamento Bibiográfico

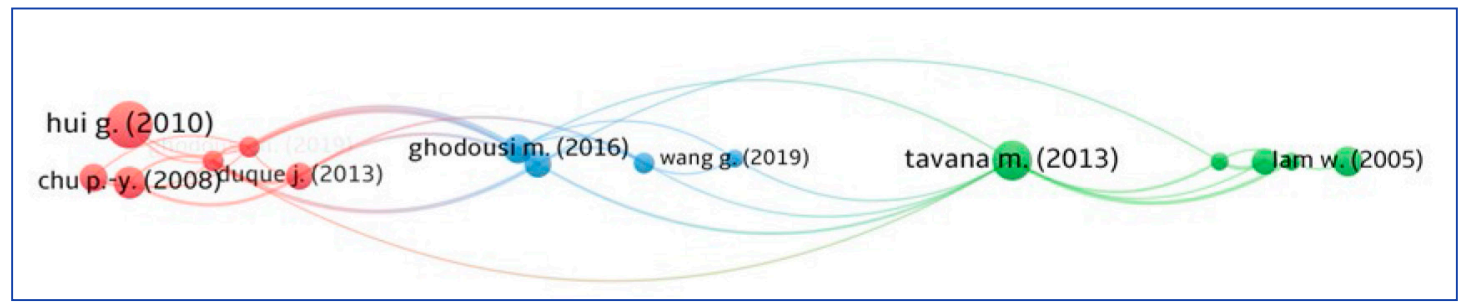

Fonte: Elaborada pelos autores.

O cluster verde é formado por artigos dedicados à criação de modelos e estruturas conceituais de CiRM. Nesse sentido, Tavana et al. (2013) avaliaram a disponibilidade e eficácia da plataforma de governo eletrônico adotada no estado de East Virginia, nos Estados Unidos. Wu (2017) avaliou a expansão do sistema de atendimento SF311, que possui call center e aplicativo na cidade de São Francisco nos Estados Unidos. Dehkordi et al. (2012) realizaram uma revisão de literatura para verificar as teorias que cercam o governo eletrônico e apontam para a existência de um arcabouço conceitual com a seguinte configuração: o contexto sociodemográfico, o processo criativo de elaboração de políticas públicas e o conteúdo das bases do governo eletrônico com indicadores de resultado e eficiência. Por sua vez, Lam (2005) traz um estudo sobre a avaliação da maturidade do governo eletrônico e aponta que quatro etapas são necessárias para consolidar a gestão da relação com o cidadão, e também sugere que países como Reino Unido e Cingapura, mesmo que avançados, ainda têm um longo caminho a percorrer no desafio da integração. Assim, o cluster verde envolve esforços de integração das estruturas e instituições que compõem a complexidade enfrentada pelos gestores públicos na prestação de serviços públicos.

O cluster vermelho compreende estudos que investigam a melhoria da relação entre governo e cidadão e o sucesso na utilização de ferramentas CiRM. Chu et al. (2008) estudaram o sistema de ouvidoria para os cidadãos de Kaohsiung e Taiwan para demonstrar os benefícios da gestão do CiRM na melhoria da compreensão do cidadão, na qualidade dos serviços públicos, na expansão da participação pública e no fortalecimento do controle. Keramati et al. (2012) estudaram os fatores críticos de sucesso na aplicação de um modelo CzRM no município de Teerã e descobriram que esses fatores de sucesso são: processo, organização, gestão do conhecimento e demandas dos cidadãos. Hui e Hayllar (2010) apontaram para a necessidade de um envolvimento genuíno da sociedade na definição dos serviços públicos e sugeriram a utilização de novas ferramentas tecnológicas, nos modelos de CiRM, para aumentar a participação do cidadão. Duque et al. (2013) conduziram pesquisa em municípios portugueses que adotam sistemas de gestão de relacionamento com o cliente e apontam que a melhoria do relacionamento com os cidadãos e uma maior qualidade da informação são alguns dos resultados mais importantes obtidos pelos municípios. 
O cluster azul traz estudos que abordam a avaliação dos serviços públicos pelos cidadãos. Ghodousi et al. (2016) realizaram uma análise de cluster utilizando o serviço telefônico 137 no Irã, e constataram que as principais demandas estavam relacionadas à qualidade do asfalto, coleta de lixo e desenvolvimento de parques. Akhondzadeh-Noughabi et al. (2013) utilizaram a técnica de mineração de texto para avaliar as informações do sistema de reclamação de serviço urbano de Teerã, revelando que esta abordagem de CiRM é útil para melhorar a gestão da perspectiva da frequência do serviço, tempo de serviço e satisfação do cidadão. Demo e Pessôa (2015) desenvolveram e validaram uma escala de relacionamento com o cidadão aplicada ao Superior Tribunal de Justiça do Brasil, inédita na literatura nacional e internacional, preenchendo uma lacuna na literatura e possibilitando futuros estudos relacionais nas áreas de marketing de relacionamento em Administração Pública.

Esses resultados mostram que a literatura de CiRM está dividida em três categorias principais: (i) o desenvolvimento de modelos integrativos e estruturas teóricas; (ii) a relação entre governo e cidadãos e os aspectos que criam essa relação; e (iii) a avaliação dos serviços públicos pelo cidadão. No entanto, não há sobreposição entre os clusters: espera-se que os clusters estejam sobrepostos e conectados. Entendemos que esta é mais uma evidência da inicipiência deste conceito. Embora os trabalhos seminais datem de 2005, CiRM é um conceito ainda emergente, conclusão que nos orientou a desenvolver uma agenda de pesquisa.

\section{Discussão e Proposta de Agenda de Pesquisa}

Os resultados desta revisão de literatura mostram que o Citizen Relationship Management - CiRM é um assunto novo na literatura, surgindo em 2005 a partir dos trabalhos de Customer Relationship Management, o CRM, uma filosofia empresarial dedicada à criação de relacionamentos de longo prazo entre organizações e clientes, com objetivo de adaptar as estratégias de CRM ao contexto das relações entre cidadãos e prestadores de serviços públicos.

A primeira discussão principal diz respeito à incipiência do assunto na literatura científica. Quando analisamos o Quadro 1 e os artigos publicados desde os trabalhos seminais de Larsen e Milakovich (2005) e Lam (2005), notamos a importância dos desenvolvimentos do governo eletrônico na pesquisa de CiRM. No entanto, não podemos entender o governo eletrônico e o CiRM como sinônimos, pois o governo eletrônico é uma das possibilidades entre as estratégias do CiRM. Nesse sentido, propomos que estudos futuros enfatizem outras estratégias de CiRM além do governo eletrônico, bem como a eficiência de tais estratégias e a combinação entre elas. Destacamos também a necessidade de desenvolvimentos conceituais sobre o CiRM para compreender a natureza desse construto, o que certamente contribuirá para o avanço teórico e empírico desse campo científico.

A segunda discussão a ser abordada é o fato de CiRM ser um construto dependente do contexto, ou seja, não apenas as práticas gerenciais no serviço público, mas a pesquisa acadêmica deve abranger a complexidade de cada contexto. A pesquisa sobre CiRM deve respeitar as particularidades de cada contexto, pois diferentes governos desenvolverão relações distintas com seus cidadãos, e a gestão dessa relação dependerá dos recursos disponíveis (pessoas, processos e tecnologias) e das características de cada cultura. No entanto, prevemos possibilidades de criação de modelos para diferentes níveis de governo, tais como local, regional e nacional, para investigar como as estratégias de CiRM são desenvolvidas nesses níveis e, mais importante, se (e como) eles estão conectados com uma estratégia de governo nacional. A pesquisa multicultural também pode ser adotada neste contexto, uma vez que diferentes locais do globo podem certamente aprender uns com os outros.

Adicionalmente, chamamos atenção para a escala desenvolvida por Demo e Pessôa (2015) no contexto brasileiro e sua capacidade de mensurar a percepção dos cidadãos sobre os serviços públicos. Acreditamos que este trabalho possibilitará diversos estudos futuros, principalmente estudos relacionais e avaliação da prestação de serviço público em diferentes variáveis importantes como a satisfação do cidadão e o desempenho do serviço público, por exemplo. Considerando que o CiRM é um construto dependente do contexto, recomendamos fortemente a validação desta escala para os diferentes países em que o CiRM foi adotado e implementado, de forma a abranger a complexidade de cada cenário e permitir que o CiRM cumpra o seu propósito de melhorar a prestação de serviços públicos.

Terceiro, analisamos as correntes de pesquisa que compõem o campo científico do CiRM. Os estudos em CiRM distribuem-se em três grandes áreas: (i) marcos conceituais e modelos, artigos que enfatizam a integração dos atores sociais e os processos geradores de estratégias de CiRM; (ii) a relação entre governo e cidadão através da utilização de ferramentas CiRM, no que se refere aos estudos que abordam os benefícios relacionais dessa relação e a dinâmica de adoção e implementação de sistemas 
de CiRM; e (iii) a avaliação dos serviços públicos pelo cidadão, no que diz respeito ao desenvolvimento de instrumentos de medição. O próximo passo é conectar tais estudos, reunindo conceituações e esforços empíricos para melhor explorar a adoção, implementação, antecedentes, consequências e limitações das estratégias de CiRM.

Também prevemos possibilidades em torno das estruturas conceituais dos sistemas de CiRM no governo eletrônico. Uma agenda importante deve considerar o CiRM não apenas como uma ferramenta de governo eletrônico, mas também como uma estratégia relacional mais ampla baseada no conceito de CRM, para o desenvolvimento e prestação de serviços públicos como resultado da interação contemporânea entre governo e cidadão. Nesse sentido, esperamos futuras investigações para explorar como os quatro estágios de maturidade do governo eletrônico de Lam (2005) interagem com outras estratégias de CiRM, e como diferentes níveis de governo (local, regional e nacional) atingem a maturidade do governo eletrônico. Além disso, recomendamos que pesquisas futuras atentem para o papel dos dispositivos digitais na formação dessas relações, entendendo o papel das mídias sociais e como o desenvolvimento de canais digitais para interagir com os cidadãos pode beneficiar tanto a sociedade quanto os gestores públicos. A adoção, implementação e eficiência de estratégias de comunicação digital devem ser mais exploradas, bem como as consequências de tais esforços para a sociedade.

A seguir, adicionamos à nossa agenda possibilidades quanto à melhoria da relação entre cidadãos e governos. Nessa perspectiva, identificamos a necessidade de explorar mais os efeitos do sistema político e do macroambiente nas estratégias do CiRM, respeitando as idiossincrasias dos aspectos culturais, jurídicos e econômicos de cada contexto de investigação. Embora o CiRM seja um conceito global, refletindo um fenômeno global, devemos entender o macroambiente, mas agir localmente, adaptando as estratégias do CiRM às necessidades das comunidades em termos de serviços públicos, enfatizando as demandas sociais mais críticas. Também sugerimos pensar nas oportunidades trazidas pelos estudos de CiRM para desenvolver práticas de estratégia no ambiente de trabalho das organizações públicas, para promover a formação e educação de funcionários públicos com foco na prestação de serviços públicos de alta qualidade e, por último, direcionar os resultados dos trabalhos de CiRM para o formulação da agenda de políticas públicas.

Em termos metodológicos, ratificamos a necessidade e oportunidade da pesquisa empírica, recorrendo a estudos multimétodos. Tais estudos possibilitam o uso da triangulação para melhorar abordagem e compreensão dos tópicos de pesquisa. Ao combinar métodos e técnicas quantitativas para medir um fenômeno, as abordagens qualitativas auxiliam na interpretação, revelando aspectos importantes não percebidos apenas pelos números.

\section{CONCLUSÃO}

O objetivo principal deste artigo foi analisar o campo científico do Citizen Relationship Management - CiRM, a fim de compreender sua estrutura intelectual e definir uma agenda de pesquisa. 0 exame de 24 artigos permitiu afirmar que o CiRM é um conceito emergente no campo científico, confirmando a necessidade de uma revisão da literatura para organizar resultados anteriores, entender o estado atual da pesquisa e definir caminhos para investigações futuras. A partir da análise da estrutura intelectual da literatura em CiRM, pudemos identificar as lacunas mais notáveis e traçar uma agenda de pesquisa, que é a principal contribuição da presente revisão de literatura. Esperamos contribuir com o desenvolvimento do corpo de conhecimentos em CiRM, orientando os esforços dos pesquisadores nas áreas de relacionamento entre governos e cidadãos; processos de adoção e implementação de CiRM; práticas de gestão pública; e eficiência na prestação de serviços públicos. Acreditamos também que nossos resultados ajudarão estudantes e pesquisadores a identificar centros de excelência e estudos de referência para desenvolver seus trabalhos e estabelecer parcerias.

Embora o presente estudo tenha usado duas importantes bases de dados, uma limitação importante deve ser abordada. Nesse sentido, notamos que a produção do CiRM não foi totalmente mapeada. Assim, se o objetivo é identificar toda a produção científica sobre o CiRM, deve-se considerar uma pesquisa mais ampla que inclua outras bases de dados e trabalhos procedentes de congressos e seminários científicos, bem como livros e teses. Além disso, como o CiRM emerge dos estudos de CRM, prevemos a possibilidade de publicações sobre a relação entre governos e cidadãos sem o uso do termo CiRM. Nesse sentido, pesquisas futuras poderiam explorar não só o CiRM, mas também a relação entre governos e cidadãos, o que, em última instância, contribuirá para o desenvolvimento do CiRM. Também sugerimos uma futura revisão da literatura sobre CiRM, a fim de acompanhar o assunto e as próximas direções de pesquisa. 


\section{REFERÊNCIAS}

Abrucio, F. L. (2007). Trajetória recente da gestão pública brasileira: um balanço crítico e a renovação da agenda de reformas. Revista de administração pública, 41(esp), 67-86.

Akhondzadeh-Noughabi, E., Alizadeh, S., Ahmadvand, A. M., \& MinaeiBidgoli, B. (2013). FTiS: A new model for effective urban management: A case study of urban systems in Iran. Cities, 31, 394-403.

Al-Khouri, A. M. (2012). Customer relationship management: Proposed framework from a government perspective times. Journal of management and strategy, 3(4), 34.

Alexandrova, A., lordanov, B., Abbas, S., Upadrasta, P., Sarasti, M. ... Hilpold, T. (2016). Systems Administration in Ontology-Based Applications: The Case of Citizen Relationship Management. International Journal of Public Administration in the Digital Age, 3(2), 37-46.

Andrade, M. M. G. (2013). Plataforma De Relacionamento Com O Cidadão: Solução de Governança Eletrônica para a Prefeitura Municipal do Salvador. PontodeAcesso, 7(2), 68-88.

Bresser-Pereira, L. C. (1996). Da administração pública burocrática à gerencial. Revista do Serviço Público, 47(1), 1-28.

Chadegani, A., Salehi, H., Yunus, M., Farhadi, H., Fooladi, M. ... Ale Ebrahim, N. (2013). A comparison between two main academic literature collections: Web of Science and Scopus databases. Asian social science, 9(5), 18-26.

Chu, P. Y., Yeh, S. C., \& Chuang, M. C. (2008). Reengineering municipality citizen electronic complaint system through citizen relationship management. Electronic Government, 5(3), 288-309.

Dehkordi, L. F., Sarlak, M. A., Pourezzat, A. A., \& Ghorbani, A. (2012). A comprehensive conceptual framework for the e-government realization. Australian Journal of Basic and Applied Sciences, 6(8), 50-64.

Demo, G., \& Pessôa, R. (2015). CRM na administração pública: desenvolvimento e validação de uma Escala de Relacionamento com o Cidadão (ERCi). Revista de Administração Pública, 49(3), 677-697.

Duque, J., Varajão, J., Vitor, F., \& Dominguez, C. (2013). Implementation of CRM systems in Portuguese Municipalities. Local Government Studies, 39(6), 878-894.

Eslami, S. (2012). A study on the customer relationship management model adaptability with the municipality services and duties environment. International Research Journal of Finance and Economics, 82, 33-48.

Fil’a, M., Schwarczová, L., \& Mura, L. (2015). Citizen satisfaction survey as a tool of citizen relationship management of local government in Slovakia. Serbian Journal of Management, 10(1), 117-129.

Fleury, S. (2005). A seguridade social e os dilemas da inclusão social. Revista de Administração Pública, 39(3), 449-467.

Ghodousi, M., Alesheikh, A. A., \& Saeidian, B. (2016). Analyzing public participant data to evaluate citizen satisfaction and to prioritize their needs via K-means, FCM and ICA. Cities, 55, 70-81.

Ghodousi, M., Alesheikh, A. A., Saeidian, B., Pradhan, B., \& Lee, C. W. (2019). Evaluating Citizen Satisfaction and Prioritizing Their Needs Based on Citizens' Complaint Data. Sustainability, 11(17), 4595.
Gupta, G., \& Aggarwal, H. (2016). Analysing customer responses to migrate strategies in making retailing and CRM effective. International Journal of Indian Culture and Business Management, 12(1), 92-127.

Hui, G., \& Hayllar, M. R. (2010). Creating public value in e-Government: A public-private-citizen collaboration framework in Web 2.0. Australian Journal of Public Administration, 69, S120-S131.

Kannabiran, G. M. J. X., Xavier, M. J., \& Anantharaaj, A. (2004). Enabling e-governance through citizen relationship management-concept, model and applications. Journal of Services Research, 4(2), 223-240.

Kavanagh, S. C. (2007). Revolutionizing constituent relationships: The promise of CRM systems for the public sector. Chicago, IL: Government Finance Officers Association.

La Falce, J. L., De Muylder, C. F., Pressot, I. M., \& Toivanen, M. A. B. L. (2014). Qualidade no serviço Público: Um estudo de caso em uma fundação pública em Minas Gerais. Amazônia, Organizações e Sustentabilidade, 3(2), 43-62.

Lara, R. D., \& Gosling, M. S. (2016a). Análise dos fatores que compõe a ouvidoria no relacionamento com o cidadão. Revista ESPACIOS, 37(23), 23.

Lara, R. D., \& Gosling, M. S. (2016b). Um Modelo De Gestão Do Relacionamento Entre Os Cidadãos E A Administração Pública. REAd. Revista Eletrônica de Administração, 22(2), 333-362.

Larsen, B. \& Milakovich, M. (2005). In International Conference on Electronic Government (pp. 57-68). Berlin, Germany: Springer.

Lytras, M. D. (2006). The Semantic Electronic Government: knowledge management for citizen relationship and new assessment scenarios. Electronic Government, 3(1), 5-17.

Payne, A. (2006). Handbook of CRM: Achieving Excelence in Customer Relationship. Oxford, UK: Elsevier.

Payne, A., \& Frow, P. (2006). Customer relationship management: from strategy to implementation. Journal of Marketing Management, 22(12), 135-168.

Peci, A., Pieranti, O. P., \& Rodrigues, S. (2008). Governança e New Public Management: convergências e contradições no contexto brasileiro. Organizações \& Sociedade, 15(46), 39-55.

Pollard, C., Young, J., \& Gregg, P. (2006). Towards a simplified framework of CRM for use in public and private sectors. Journal of Information Technology Case and Application Research, 8(2), 24-38.

Ponte, V. (2015). CiRM: CRM no Setor Público. In G. Demo (Org.), Marketing de Relacionamento \& Comportamento do Consumidor (pp. 137-174). São Paulo, SP: Atlas.

Schellong, A. (2008). Citizen Relationship Management. A Study of CRM in Government. Bern, Switzerland: Peter Lang.

Secchi, L. (2009). Modelos organizacionais e reformas da administração pública. Revista de Administração Pública, 43(2), 347-369.

Shaikh, R., \& Khan, M. R. (2014). Citizen Relationship Management: A Decisive Parameter of G2C e-Governance Web Portals of Maharashtra India. International Journal on Recent and Innovation Trends in Computing and Communication, 2(8), 2234-2239. 
Sukarno, G., Azizah, N., Nawangsari, E. R., Izaak, W. C., \& Farida, S. N. (2020). Citizen relationship management (CnRM) to build the awareness of anti-corruption: Collaborative governance perspective. International Journal of Psychosocial Rehabilitation, 24(8), 1299-1306.

Tavana, M., Zandi, F., \& Katehakis, M. N. (2013). A hybrid fuzzy group ANP-TOPSIS framework for assessment of e-government readiness from a CiRM perspective. Information \& Management, 50(7), 383-397.

Templier, M., \& Paré, G. (2015). A framework for guiding and evaluating literature reviews. Communications of the Association for Information Systems, 37(1), 112-137.

Tranfield, D., Denyer, D., \& Smart, P. (2003). Towards a methodology for developing evidence-informed management knowledge by means of systematic review. British journal of management, 14(3), 207-222.
Twizeyimana, J. D., \& Andersson, A. (2019). The public value of E-Government-A literature review. Government Information Quarterly, 36(2), 167-178.

Vaerst, T., Steffens, T., \& Lokaiczyk, R. (2015). Concerns Management, E-Government and E-Participation: Experiences and Findings from Germany. International Journal of E-Planning Research, 4(4), 36-49.

Van Eck, N. J., \& Waltman, L. (2017). Citation-based clustering of publications using CitNetExplorer and VOSviewer. Scientometrics, 111(2), 1053-1070.

Zamanian, M., Khaji, M. R., \& Emamian, S. M. S. (2011). The value chain of citizen relationship management (CzRM): A framework for improvement. African Journal of Business Management, 5(22), 8909-8917.

Zupic, I., \& Čater, T. (2015). Bibliometric methods in management and organization. Organizational Research Methods, 18(3), 429-472.

Daniel Carvalho

ORCID: https://orcid.org/0000-0002-5307-7680

Doutorando em Administração pelo Programa de Pós-Graduação em Administração da Universidade de Brasília (UnB). E-mail: danizalho@gmail.com

Gisela Demo

ORCID: https://orcid.org/0000-0003-1864-0471

Doutora em Psicologia Social do Trabalho e Organizações pela Universidade de Brasília (UnB); Professora do Programa de Pós-Graduação em Administração da Universidade de Brasília (UnB). E-mail: giselademo@gmail.com

Júlio Medeiros

ORCID: https://orcid.org/0000-0002-1208-6740

Mestre em Administração de Empresas pela Universidade de Brasília (UnB). E-mail: julioelpidio@gmail.com

Fernanda Scussel

ORCID: https://orcid.org/0000-0001-7953-6710

Doutora em Administração pela Universidade Federal de Santa Catarina (UFSC). E-mail: contatofernandascussel@gmail.com 